\title{
The Oseberg transition: visualization of global bifurcations for the Kuramoto-Sivashinsky equation
}

\author{
M. E. Johnson*, M. S. Jolly†, and I. G. Kevrekidis ${ }^{\ddagger}$
}

December 9, 1999

\begin{abstract}
We present and discuss certain global bifurcations involving the interaction of one- and two-dimensional invariant manifolds of steady and periodic solutions of the Kuramoto-Sivashinsky equation. Numerical bifurcation calculations, dimensionality reduction using approximate inertial manifolds/forms, as well as approximation and visualization of invariant manifolds are combined in order to characterize what we term the "Oseberg transition".
\end{abstract}

\section{Introduction}

The one-dimensional Kuramoto-Sivashinsky equation (KSE) with periodic boundary conditions can be written in the following form:

$$
\begin{aligned}
u_{t}+4 u_{x x x x}+\alpha\left[u_{x x}+u u_{x}\right] & =0, \\
u(t, x) & =u(t, x+2 \pi) .
\end{aligned}
$$

It arises in several physical contexts as an amplitude equation for spatiotemporal growth of instabilities (flame fronts [32], reaction-diffusion problems [25] as well as thin film flow down an inclined plane [5]). Pioneering computational work in the early 1980s [16] established it as a model equation for spatiotemporal pattern formation, and breakthroughs in the theory of inertial manifolds [10], as well the first numerical efforts at their implementation as approximate inertial manifolds [9] also employed it as their par excellence example. The discovery of persistent heteroclinic cycles for the KSE [23] made it also a representative example for the study of bifurcations with symmetry ([1], [22], [7], [3]).

\footnotetext{
${ }^{*}$ Institute for Mathematics and its Applications, University of Minnesota, Minneapolis, MN 55455, USA

†Department of Mathematics, Indiana University, Bloomington, IN 47405, USA

$\ddagger$ Program in Applied and Computational Mathematics, Princeton University, Princeton, NJ 08544, USA
} 
This combination of physical motivation, rich spatiotemporal behavior, the importance of symmetries and the existence of inertial manifolds have established the KSE as a workhorse example for the study of complex dynamics in dissipative partial differential equations (PDEs). A lot is known about its detailed "low $\alpha$ " bifurcation diagrams (e.g. $[23,31,4,3]$ ) and extensive research is carried out in trying to understand the nature and scalings of the spatiotemporal chaos it exhibits at large values of $\alpha[6,36]$

In this paper we will describe a particular global bifurcation exhibited by the KSE. This transition involves both one- and two-dimensional invariant manifolds of steady as well as periodic solutions of the PDE; bifurcation calculations as well as invariant manifold approximation and visualization are crucial in the presentation and the understanding of what occurs. The low-dimensionality of the dynamics is vital in this exposition, in order to make the low-codimension stable manifolds of saddle-type steady states and limit cycles visible in infinite dimensional Hilbert spaces. This is accomplished using an approximate inertial form and restricting to invariant subspace of odd functions to reduce the longterm dynamics of the PDE to those of a system of three ordinary differential equations, for which the stable manifolds of interest are of dimension one and two.

A partial exploration of the bifurcation we study has appeared in a recent paper by Ponce-Dawson and Mancho [7]. In their study of heteroclinic cycles in the KSE with periodic boundary conditions, using an approximate inertial form similar to the one used here, they noticed and analyzed many of the phenomena constituting the transition picture we will see here. The ability to approximate and visualize two-dimensional invariant manifolds allows us, in this paper, to show elements of the transition which were correctly deduced from simulations but not actually seen in [7], along with additional phase space/parameter space structures completing the bifurcation picture.

The paper is organized as follows: In section 2 we give concise numerical evidence (using a sufficiently converged discretization of the PDE) suggestive of the phenomenon we will proceed to analyze. An informal pictorial terminology of the elements involved is also introduced at this stage. There follows, in Section 3, a brief discussion of the reduction of the PDE to a three-dimensional approximate inertial form, a step which is essential for the visualization of the relevant global bifurcations; the development (through bifurcation calculations and continuation) of the basic "players" in the transition is presented here. The main results are in Section 4, which contains a sequence of numerical experiments exploring the bifurcation as well as what we consider our best attempts at its visualization. We conclude with a brief discussion of the results.

\section{The Oseberg Transition}

We first describe the global transition in question in a relatively high-dimensional phase space, representative of the full PDE. We restrict our exploration to the invariant subspace of odd functions, in which case the solutions may be repre- 
sented by the Fourier sine series

$$
u(t, x)=\sum_{j=-\infty}^{\infty} u_{j}(t) e^{i j x}=\sum_{j=1}^{\infty} b_{j}(t) \sin (j x),
$$

where the reality and oddness conditions on $u$ give

$$
u_{-j}=\bar{u}_{j} \quad \text { and } \quad u_{j}=-i b_{j} / 2 .
$$

An eight-mode Galerkin truncation of the PDE is sufficiently converged to be qualitatively and quantitatively accurate in the parameter regime we study [23].

Figure 1 shows the dramatic qualitative change the global attractor undergoes as the bifurcation parameter $\alpha$ passes through a certain critical value $\alpha_{\mathrm{O}} \approx 32.9$. Figure $1 \mathrm{a}$ is taken "before" $\left(\alpha<\alpha_{\mathrm{O}}\right)$, and Figure $1 \mathrm{~b}$ "after" the global bifurcation.

To describe this transition further, it is useful to first examine in Figure 1c a "skeleton" of the global attractor consisting of five steady states, certain one-dimensional unstable manifolds and a limit cycle (which we refer to as $\left.\gamma_{\text {Hopf }}\right)$. The axes in this figure are the first (horizontal, $\sin 1 x$ ), second (vertical, $\sin 2 x$ ) and third (into the plane of the picture, $\sin 3 x$ ) Fourier coefficients of the solution. These steady states, for reasons that will be explained below, carry the shorthand characterizations the origin (at the origin of the plotting coordinates), the top and bottom bimodals (on the upper and lower part of the vertical axis respectively) and the two mixed mode bi-tris, off-axis.

For the parameter value corresponding to this figure (and the nearby parameter range), the hyperbolic steady states maintain a constant stability type (the origin: saddle, two (real) unstable eigenvalues; top bimodal: stable; the two bi-tris: saddles, one unstable eigenvalue; bottom bimodal: two (complex) unstable eigenvalues). Using the figure as a visual guide, we term "upward-" and "downward-pointing" the two sides of the one-dimensional unstable manifolds of the bi-tri states. The upward-pointing sides of both bi-tri unstable manifolds asymptotically approach the top bimodal. The asymptotic fate of the downward-pointing sides of these two manifolds is, as we will see, sensitive to changes in the parameter. In this figure one of these two manifolds is only partially plotted, so as not obscure the picture; the other one can be obtained through symmetry. These one-dimensional manifolds form part of the boundary of the two-dimensional unstable manifold of the origin, the majority of which is rendered in yellow in Figures 1a,b. In Figure 1a the two-dimensional unstable manifold of the bottom bimodal (in red) is topologically an open disk, the boundary of which is the limit cycle $\gamma_{\mathrm{Hopf}}$.

There are two attractors in this picture: the top bimodal, and the stable limit cycle $\gamma_{\text {Hopf }}$. The basin boundary separating their corresponding basins of attraction cannot be visualized in this eight-dimensional phase space; it is a codimension-one manifold (a pair of them, really), the stable manifold(s) of the bi-tri state(s). Notice that in Figure 1b, the red manifold (the two-dimensional unstable manifold of the bottom bimodal, which before the critical value $\alpha_{O}$ 
approached asymptotically the $\gamma_{\text {Hopf }}$ limit cycle) has "burst through" this invisible basin boundary and now approaches asymptotically both $\gamma_{\text {Hopf }}$ and the top bimodal. The pair of codimension-one bi-tri stable manifolds, which play a vital role in the transition we are witnessing, cannot be visualized in this threedimensional projection of the eight-dimensional phase space. Indeed, the computation of a manifold of such high dimension is beyond the current state of the art. These two two-dimensional unstable manifolds (of the origin, in yellow, and of the bottom bimodal, in red), along with all other two-dimensional (un)stable manifolds we will follow in this work, were computed as in [18]. Briefly this method evolves a discrete (and adaptive) collection of points, initially taken to lie on the unstable eigenspace of the steady state in question, into a skeleton of the approximation to the manifold. For alternative, more recent approaches to computation of two-dimensional (un)stable manifolds, see [24].

In what follows we will need to make repeated references to many of the particular phase space elements described above. The standard terminology (for example, "two-dimensional stable manifold of steady state A", and "upward pointing side of the one-dimensional unstable manifold of steady state B"), make the description, in our opinion, awkward. On a slightly playful note, we decided that our global attractor bears an uncanny resemblance to a sailboat (indeed when we first saw the transition between Figures $1 \mathrm{a}$ and $\mathrm{b}$, we began to refer to it as the "cup-boat transition"). In Figure 1a, the attractor is much like a dinghy, i.e. a small, deckless sailboat, and in Figure 1b, it has been transformed into a Viking ship, in particular, one like that recovered in the archaeological dig in Oseberg, Norway in 1903 [2],[15] (see Figure 2). We will therefore take advantage of this similarity, and borrow a bit of nautical terminology. Henceforth, we will refer to the two-dimensional unstable manifold of the bottom bimodal as the hull, the two-dimensional unstable manifold of the origin as the sail, the segment of the invariant $\sin (2 x)$-axis between the two bimodals as the mast, and the onedimensional unstable manifolds of the bi-tris as the ropes. In fact these ropes lie in the boundary of the sail, due to transversality and the $\lambda$-lemma [28],[17]. We believe this imagery makes the narrative more succinct; we hope our readers will not bristle at the informality, and adopt our naming this bifurcation "the Oseberg transition".

\section{Dimensionality Reduction}

Reduction of the dimension of the phase space is essential for visualizing and, hopefully, understanding the geometry of the global bifurcations witnessed above. A first step in the reduction (halving the dimension) has already been accomplished through restriction of our observations to the invariant subspace of odd functions. We carry the next, crucial reduction step, by means of an approximate inertial manifold (AIM). The existence of exact inertial manifolds (along with estimates of their dimensions) has been well established for the KSE [10], [35],[29],[21]. In addition, the effectiveness of three-dimensional AIMs in capturing the "correct" long-term dynamics over the parameter range of interest 
here, is also well-documented, at least as far as the local bifurcations, leading to the skeleton of the global attractor described above $[9,19]$ is concerned. The restriction of the flow to such a three-dimensional manifold is described by a system of three ordinary differential equations (an approximate inertial form, or AIF). It is the dynamic behavior of this system that we study in detail in the sections that follow.

To describe the AIM we express the KSE as an evolutionary equation

$$
\frac{d u}{d t}+A u+R(u)=0, \quad u \in H
$$

where $H$ is an appropriate Hilbert space (see [34]). The linear operator $A$ may be taken to be that given by $A u=u_{x x x x}$ along with periodic, odd boundary conditions. The remaining terms are then collected in $R$. The infinite-dimensional phase space $H$ is split into low- and high-wavenumber modes by means of the projectors

$$
P: H \rightarrow \operatorname{span}\{\sin (x), \sin (2 x), \sin (3 x)\}, \quad Q=I-P .
$$

The particular AIM used here can be found as the second iterate of a contraction mapping indicated by the sequence of explicit functions

$$
\begin{aligned}
\Phi_{j+1}(p) & =-A^{-1} Q R\left(p+\Phi_{j}(p)\right), \quad j=0,1, \ldots \\
\Phi_{0}(p) & \equiv 0, \quad p \in P H .
\end{aligned}
$$

The associated approximate inertial form studied here is

$$
\frac{d p}{d t}+A p+P R\left(p+\Phi_{2}(p)\right)=0 .
$$

In contrast, the standard Galerkin approximation can be expressed as

$$
\frac{d p}{d t}+A p+P R(p)=0 .
$$

An explicit formulation of both the AIF 7 and the Galerkin approximation can be found in the appendix in [19].

There is considerable theoretical justification for using $\Phi_{2}$ as an AIM. The graph of the limit $\Phi_{\infty}$ (an implicit relation) of the sequence in (6) is a manifold containing all steady states of the PDE. Early estimates showed that the distance from the attractor to the manifold given by the second iterate $\operatorname{graph}\left(\Phi_{2}\right)$, is of the same order as to that given by $\Phi_{\infty}$, and that in both cases this distance is smaller than that of the trivial (flat) manifold described by $\Phi_{0}$ [19]. Subsequent work has revealed however, that this advantage for the AIMs amounts to an algebraic improvement on top of an error which decays exponentially with the dimension of the manifold, due to Gevrey regularity of the solutions [26]. Thus the fact that, in computations, the 3-mode AIF (7) seems to capture the correct long-term dynamics while the 3 -mode Galerkin approximation does not, can be explained by the low dimension at which the comparison is made. We 
should emphasize that the choice for the dimension (three) is based on the desire to visualize global interactions in phase space, and that this choice is to some extent, vindicated by computational evidence. Ponce-Dawson and Mancho [7] used a slightly modified version of this AIF, in which the highest order terms were dropped. While qualitatively similar, their bifurcations are slightly shifted in parameter and phase space from ours.

We do not, in fact, know that an exact, three-dimensional inertial manifold exists for the KSE over the parameter range of interest here. Roughly speaking, the existence of an inertial manifold can be established if a gap in the spectrum of $A$ dominates, in a specific way, the Lipschitz constant of the nonlinear term. This requires that the equation be prepared; the nonlinear term is truncated beyond a ball in phase space. If the ball of truncation is absorbing, then it contains the attractor of the PDE, and as far as the dynamical behavior is concerned, such a preparation is simply a mathematical device. It is shown in [21] that for all $\alpha<36$, truncating at a rigorous absorbing ball yields an inertial manifold for the KSE of dimension under 200. It is also shown in [21] however, that truncating at a smaller ball, yet one which still contains the attractor as we know it from computational evidence, provides a five-dimensional inertial manifold for all $\alpha<36$, for this (perhaps) "overly-prepared" version of the KSE. So the latter estimate, which could not a priori have been expected to be so sharp, actually comes quite close to justifying the use of a three-dimensional system over the parameter range of interest here.

\section{Symmetries and invariant subspaces}

A brief discussion of certain symmetry elements of the PDE may be of assistance in the description of the solutions involved in our transition and their interactions. Consider the KSE PDE with periodic boundary conditions (not just odd), with the corresponding change of $H$ in (4). Any spatial translation of a solution $T_{\theta}: u(t, x) \rightarrow u(t, x+\theta)$ is also a solution, as is the result of the transformation $S: u(t, x) \rightarrow-u(t,-x)$. These two actions generate the group $O(2)$. The fact that $T_{\theta}$ and $S$ commute with the nonlinear term $R$ for the KSE, implies that the Galerkin approximation in (8), and the AIF in (7) also enjoy $O(2)$ symmetry.

Let $G$ be a subgroup of $O(2)$ and let $\operatorname{Fix}(G)$ denote the subspace of $H$ (or $P H)$ consisting of elements left fixed by $G$. Then $\operatorname{Fix}(G)$ is an invariant subspace [13],[14] for the PDE (or its finite-dimensional approximations (8), (7)). In particular, the subspaces consisting of $2 \pi / k$-periodic functions are invariant for each integer $k$, since these are precisely the functions left unchanged by actions of $T_{2 \pi / k}$. Note also that $\operatorname{Fix}(S)$ is the subspace of odd functions.

Though the restriction to the invariant subspace of odd functions destroys the $O(2)$-symmetry, the intersection of $\operatorname{Fix}\left(T_{2 \pi / k}\right)$ with the odd subspace is still invariant. Since in terms of Fourier coefficients

$$
T_{2 \pi / k}: u_{j} \rightarrow e^{i j 2 \pi / k} u_{j},
$$

it is easy to see that the $\sin (2 x)$-axis and the $\sin (3 x)$-axis are also invariant for 
the AIF ( 7 ) since we use only the first three modes $u_{1}, u_{2}, u_{3}$. Finally, we remark that the $T_{\pi}$ symmetry for our three-dimensional reduced system corresponds to the transformation

$$
g:\left(b_{1}, b_{2}, b_{3}\right) \rightarrow\left(-b_{1}, b_{2},-b_{3}\right),
$$

a symmetry which is identical to that found in the Lorenz equations [27].

\section{Observed phenomena}

\subsection{Setting the stage}

We start with a brief description of how the basic "players" in the global attractor in Figure 1 arose through the now well-established sequence of low- $\alpha$ bifurcations of the KSE. This serves not only to set the transition we are studying in the context of other known KSE bifurcations, but also to reiterate the number of unstable eigenvalues (and therefore invariant manifold dimensions and codimensions) for each of the "players".

Figure 3 shows the bifurcation diagram, with respect to $\alpha$, of the approximate inertial form (7). This is in good qualitative (and reasonable quantitative) agreement with the corresponding diagram for the PDE (obtained through large converged truncations [19],[23]). The trivial solution is stable (three negative real eigenvalues) for $\alpha<4$ and loses one stable eigenvalue in each of three successive bifurcations as $\alpha$ increases. The first bifurcation (a pitchfork in the subspace we study) to nonuniform steady states occurs at $\alpha=4$. The resulting steady states have been termed unimodal as they initially possess one spatial hump; the eigenvector of the critical eigenvalue is $(1,0,0)$, and they are therefore born "in the $\sin (x)$-direction". Due to symmetry, both unimodal branches appear superposed in the diagram. Two replicas [31] of this bifurcation also appear in the diagram (one for bimodal solutions at $\alpha=16$, and one for trimodal solutions at $\alpha=36$ ).

One of the two bimodal branches undergoes (at $\alpha=\alpha_{b t} \approx 22.0$ ) a pitchfork bifurcation to a pair of bi-tri branches, and subsequently (at $\alpha=\alpha_{H} \approx 30.2$ ) a Hopf bifurcation to a limit cycle, $\gamma_{\text {Hopf }}$, which possesses a particular spatiotemporal symmetry. In terms of the PDE this symmetry is described by

$$
u(t, x) \rightarrow u(t+\tau / 2, x+\pi),
$$

and in terms of the three-dimensional states we are dealing with here

$$
\left(b_{1}, b_{2}, b_{3}\right) \rightarrow\left(-b_{1}, b_{2},-b_{3}\right) \text { and } t \rightarrow t+\tau,
$$

where $\tau$ is the period in each case. Specifically, the limit cycle, as a set, is invariant under the action of the symmetry $g$ (as defined in (9)).

Throughout the parameter regime of interest, the bi-tri steady states possess one unstable and two stable eigenvalues. The upward-pointing sides of the bi-tri unstable manifolds (ropes in our nautical terminology) asymptotically approach the top bimodal. The downward-pointing ropes, for values of $\alpha$ sufficiently close 
to (and larger than) $\alpha_{b t}$, asymptotically approach the bottom bimodal or (later, after $\alpha_{H}$ ) the limit cycle $\gamma_{\text {Hopf }}$. For larger values of $\alpha$ the long-time fate of the downward ropes can become, as we will see, quite sensitive to the parameter. We provide in Figure 4 a sequence of phase portraits at several values of $\alpha$.

For values of $16<\alpha<36$, between the bimodal and trimodal bifurcations, the origin has a two-dimensional unstable manifold (the sail). The bi-tri stable manifolds (the tent $t^{1}$ ) are also two-dimensional and are observed to intersect the sail transversely throughout the parameter regime we study. The term tent is perhaps more justified in Figure 5, which shows two distinct views of these two, two-dimensional stable manifolds: the blowup, close to the origin, of the two manifold segments shows a distinct fold similar to the ridge of a pup tent. This tent corresponds to the pair of codimension-one stable manifolds whose collision with the hull at $\alpha=\alpha_{\mathrm{O}} \approx 32.6$, transforming the dinghy into the Viking ship, constitutes the Oseberg transition for the AIF (7).

The transitions we describe are, we believe, qualitatively correct; the exact parameter values at which they occur are not so easy to pinpoint through our numerical procedures. Indeed, invariant manifold approximations may be (in the neighborhood of global bifurcations) particularly sensitive to the parameter values, to the size of initial local manifold approximations, as well as the time step size and accuracy of the numerical integrator used to evolve the manifold approximation. These errors in the actual critical parameter value are, however, not qualitatively important (they are subsumed by the approximation made using an AIF as opposed to a converged discretization of the PDE). Similar phenomena occur (at nearby parameter values) for different AIFs, different manifold approximations, and different integrators; in that sense, we believe that the Oseberg transition is robust.

Before continuing the study of this transition, we describe the phase space in further detail for $\alpha$ smaller than $\alpha_{\mathrm{O}}$. The hull of the dinghy is initially (just after $\alpha_{H}$ ) composed of trajectories which are asymptotically attracted to the limit cycle $\gamma_{\text {Hopf }}$; this limit cycle acts as this hull's rim (as was already suggested in Figure 1a). Near the Hopf bifurcation the two stable Floquet multipliers of the limit cycle are real, and the rim of the hull is much like that of a bowl. As $\alpha$ increases, the multipliers collide $(\alpha \approx 31.5)$ become complex, travel around zero inside the unit circle, and eventually coalesce and split on the positive real axis. When the multipliers of $\gamma_{\text {Hopf }}$ are complex, the hull spirals around its rim. Since the symmetry $g$ composed with itself gives the identity $\left(g^{2}=I\right)$, it is not surprising that the multipliers do not split following their collision on the negative real axis [33]. After the multipliers collide on the positive real axis and split, they remain real as $\alpha$ changes, and one of them passes through the unit circle at +1 in a symmetry-breaking bifurcation at $\alpha \approx 32.7$ giving rise to a pair of linked asymmetric stable periodic solutions (each is the image of the other under the symmetry $g$ ). As a pair of curves, these asymmetric limit cycles have linking number [11] equal to one, as does the combination of either asymmetric

\footnotetext{
${ }^{1}$ Interestingly, a number of tents were also found at the archeological dig which uncovered the fully intact Oseberg Viking ship.
} 
limit cycle with the Hopf orbit.

It is interesting to consider the shape of the hull as it approaches its rim after the multipliers have split and become real again (it still spirals) and after the symmetry breaking bifurcation giving rise to the two asymmetric limit cycles (it both spirals and asymptotically approaches these two new "rims"). Figure 7 shows the Moebius band that constitutes both sides of the two-dimensional unstable manifold of $\gamma_{\mathrm{Hopf}}$ after the symmetry breaking bifurcation. The interaction of this band with the hull close to the rim will not be further pursued here; other, more dramatic phenomena affect the hull.

Numerical continuation of both the symmetric and the asymmetric limit cycle branches is known to result in a multitude of homoclinic and heteroclinic scenarios [4], [7]. Figure 6 shows two views (one of them a blowup) of these scenarios, along with representative shapes of the hetero/homoclinic orbits which they asymptotically approach. These scenaria, however, occur at values of $\alpha$ considerably larger than $\alpha_{\mathrm{O}}$ (see captions in Figures 6 and 14). We will provide evidence below indicating that additional homoclinic and heteroclinic connections are implied by the Oseberg global transition, and identify some of the (different) families of limit cycles associated with these additional connections.

\section{2 Šilnikov orbits}

The ropes alone give an indication of a global bifurcation. In Figure 8, the ropes approach the rim of the hull as $t \rightarrow \infty$, for $\alpha=32.62666$, while at $\alpha=32.62667$, after an excursion near the rim of the hull, they pass near the bi-tri en route to the stable bimodal state. This is suggestive of a Šilnikov (double) heteroclinic connecting orbit. A similar (double) heteroclinic connection was reported in [7] around what corresponds to $\alpha=32.56$. We recall that the highest order terms were dropped in the AIF used in [7], which most probably explains this discrepancy. We expect that, in phasexparameter space, branches of limit cycles will asymptotically approach these connections (as their periods tend to infinity). One may expect both symmetric limit cycle branches (asymptotically approaching double heteroclinic connections) as well as asymmetric limit cycle branches (asymptotically approaching homoclinic connections between each bi-tri and itself). One can further classify these closed curves (both the "infinite period" ones and the limit cycle branches which asymptote to them in phase $x$ parameter space) by the number of times they wrap about the $\sin (2 x)$-axis, an invariant line which cannot be crossed. A period- $k$ orbit would be one which wraps around this invariant line $k$ times. This classification is of significance since a period$2^{n_{1}} \cdot k$ limit cycle can be born only from a limit cycle of period $2^{n_{2}} \cdot k$, for certain combination of integers $n_{1}$ and $n_{2}$; similarly, an infinite-period connecting orbit must have this same "signature" as the finite-period limit cycles that asymptotically approach it.

We found it particularly intriguing that this Silnikov orbit occurs close (in parameter space) to our best estimate of the Oseberg transition critical value, $\alpha_{\mathrm{O}}$. The remainder of this paper is devoted to describing a mechanism which 
links these two seemingly independent global bifurcations: the heteroclinic connections between the bi-tris, and the escape of the hull towards the top bimodal.

A close examination of a cross-section of the hull reveals the transformations it undergoes as we approach the Oseberg transition. We compute such a crosssection by taking a large number of initial conditions on the linear approximation (near the bottom bimodal) to the hull and then iterating the return map defined on the Poincaré section $b_{1}=0$. In Figure $9(\mathrm{~b}-\mathrm{i})$, the hull becomes increasingly complicated as higher-period structures emerge within its rim. The result of following the flow of such a set of initial conditions in the full phase space at $\alpha=32.58$ indicates the presence of an attracting period-3 limit cycle (see Figure $9(\mathrm{a})$ ); this solution coexists with the symmetric limit cycle $\gamma_{\mathrm{Hopf}}$, and, as a matter of fact, the hull asymptotically approaches both of them.

Where do these new periodic solutions (also found in [7]) originate? Linearizing at the bi-tri steady states, we find numerically that the (real) unstable eigenvalue is over three times the magnitude of the real part of the stable eigenvalue conjugate pair over the range $32 \leq \alpha \leq 33$. In a situation such as this, where the rate of repulsion exceeds that of attraction, the existence of a Šilnikov orbit implies that at the critical parameter value, there is a sequence of limit cycles with longer and longer periods which approaches the Šilnikov orbit in phase space [12]. Such limit cycles typically lie on a single branch in parameter $\times$ phase space which undergoes an infinite number of saddle-node bifurcations as it switches back and forth from left to right in a bifurcation diagram (see Figures 3, 6) An even closer look at the Poincare cross-section of the hull at in Figure 10(a) indeed shows the result of such a period-3 saddle-node bifurcation: a period-3 saddle limit cycle along with its unstable manifold. One side of the (in section, one-dimensional, in the full phase space, two-dimensional) unstable manifold of this saddle limit cycle approaches our familiar, still stable, symmetric $\gamma_{\mathrm{Hopf}}$. The other side of this unstable manifold, on the Poincaré map, approaches a stable period-3 limit cycle, which was born in the same saddle-node bifurcation as the saddle period-3, and which, in Figure 10(a), is visually indistinguishable from the saddle at this magnification and value of $\alpha$. Also shown in Figure 10(a) are both branches of the stable manifold of the period-3 saddle limit cycle (again, two-dimensional in the full phase space but one-dimensional in the Poincaré section).

Figure 10(b) is our most important computational result. It shows the same elements, but with the cross-section of the hull superposed. It is clear that the stable manifold of the period-3 saddle intersects the hull transversely, implying the existence of structurally stable connecting orbits between the bottom bimodal and the saddle period-3, even upon the inception of the saddle-period-3 at a saddle-node bifurcation. We provide in Figure 11 a schematic version of a wedge of the hull together with the stable manifolds of the period-3 orbits. Figure 10(c) shows the same objects but at a slightly larger value of $\alpha$ along with yet another periodic orbit arising in a saddle-node bifurcation: a period- 5 saddle limit cycle located near the "tips" that the hull section develops. A small portion of the stable manifold (looking much like a slash through each triangle) of the saddle period-5 is also shown in Figure 10(c) indicating transversality in 
this case as well. Figure 12 shows the results of numerical continuation of various such periodic solutions, which appear and often disappear in saddle-node bifurcations, using AUTO [8]. In Figure 12(a), the period-3 solutions as well as the $\gamma_{\text {Hopf }}$ are shown to eventually asymptotically approach Šilnikov orbits. In [7] the period-3 solutions were found to undergo a period doubling sequence of bifurcations to apparently chaotic dynamics. A closer look at the period-5 and associated period-10 branches in Figures 12(c-d) does not indicate a Šilnikov bifurcation, yet this continuation is by no means exhaustive. While none of these branches of periodic orbits seem to approach the Šilnikov connecting orbit at $\alpha \approx 32.63$ (refer back to Figure 8), we expect that there exist additional isolated branches of periodic orbits which do.

In summary: periodic solutions, born at saddle-node bifurcations, are found (as expected) close to the value of $\alpha$ at which a (double) heteroclinic connection develops between the two bi-tri states. We do find several families of such periodic solutions close to the value of $\alpha$ at which the double heteroclinic connection between the two bi-tri steady states occurs. The parameter dependence of many of these periodic solutions is strongly suggestive of their participation in a Šilnikov type connection scenario.

The important element is that all these solutions are "born" with their stable manifolds already tangled with the two-dimensional unstable manifold of the bottom bimodal (the hull). It is therefore not surprising that, as these periodic solutions approach a (double) heteroclinic orbit -in other words, as they approach the ropes and the tent, the stable manifold of the bi-tri states-, the hull, which contains orbits asymptoting to them, also approaches the heteroclinic orbit -and thus the tent, the two-dimensional stable manifold of the bi-tri states-. It then makes sense that even before (in parameter space) the basic double heteroclinic orbit connecting the two bi-tri states occurs, the two-dimensional unstable manifold of the bottom bimodal interacts with the two-dimensional stable manifold of the bi-tris, in the heteroclinic explosion that creates the Oseberg ship.

We review the sequence of connections: the hull is tangled with saddle periodic orbits; periodic orbits asymptote to the ropes; the ropes coincide with the tent at the heteroclinic value. We therefore expect that the hull approaches the tent (and interacts with it) before (in parameter space) any given periodic branch asymptotes to the (double) heteroclinic connection.

\section{Conclusion}

We presented a computer-assisted study of a particular global transition, involving invariant manifolds of several steady states and limit cycles; these manifolds have dimension (or codimension) one and/or two in our study. Model reduction allowed the visualization of the low codimension invariant manifolds. Through this study we were able to link two distinct global bifurcations: a double heteroclinic connection between two symmetric steady states, and the Oseberg transition, the intersection of the stable manifold of these states with the two-dimensional unstable manifold of a third, unstable steady state. The 
missing link was provided by limit cycle families, related with the Šilnikov double heteroclinic connection, but who are also (through their stable and unstable manifolds) tangled with the two-dimensional unstable manifold in question. The connection of these two, in principle unrelated, events justifies the numerical observation of structurally stable heteroclinic cycles in the full (periodic, non-odd) PDE close to the parameter value at which the double heteroclinic connection of the bi-tri states occurs [7]. The picture is certainly far from complete; all this is just the onset of the complex, gradual interaction of the unstable manifold of the bottom bimodal with the bi-tri steady states, the various primary $\left(\gamma_{H o p f}\right.$, asymmetric) and secondary (period-3, $-5,-10$ and more) limit cycles and their stable manifolds. Hopefully the information presented here will motivate the study of the dynamics in the neighborhood of the bi-tri steady states close to this transition.

There is no guarantee that the mechanism behind the Oseberg transition described here by the use of a three-dimensional AIF, is in fact consistent with the way the transition takes place for the PDE, or for that matter, the eightmode Galerkin approximation used to produce Figure 1. The reduction to a three-dimensional system was, however, essential for the visualization of stable manifolds of low codimension. The AIM used in (7) is only a rough approximation to the global attractor, (and to an exact inertial manifold, should one of such low dimension exist). Since the computations for this paper have been carried out, a new algorithm for computing inertial manifolds to arbitrary accuracy has been developed in [30]. The manifold produced by the latter algorithm was demonstrated in [20] to be considerably more accurate than the AIM in (7), when tested at a small sampling of points on the global attractor of the KSE. Further confirmation that the mechanism described here is as for the PDE, could thus, in principle, be obtained by repeating the visualization study presented here, but using a more accurate approximation to an inertial manifold. We believe, however, that the computations presented here, as well as computations of low-dimension (as opposed to low-codimension) invariant manifolds, like the hull, in large PDE truncations, provide convincing evidence the essential phenomenology we present is still valid in the PDE.

\section{Acknowledgments}

We thank the National Science Foundation for continuing support over the years we have been exploring the dynamics of the KSE. During that period, good portions of that effort came while all three authors enjoyed the hospitality of both the Center for Nonlinear Studies at the Los Alamos National Laboratory and the Institute of Mathematics and its Applications at the University of Minnesota. This work constitutes a part of the Ph.D. Thesis of MEJ in the Program in Applied and Computational Mathematics at Princeton University. This paper is dedicated to the Torrey Pines Foundation which, for many years and in many ways, has supported the research of IGK and MSJ. 


\section{References}

[1] D. Armbruster, J. Guckenheimer, and P. Holmes. Kuramoto-Sivashinsky dynamics on the center-unstable manifold. SIAM J. Appl. Math., 49(3):676-691, 1989.

[2] A.W. Brogger and H. Shetelig. The Viking Ships. Twayne, New York, 1971.

[3] H. S. Brown and I. G. Kevrekidis. Modulated traveling waves for the Kuramoto-Sivashinsky equation. In Pattern formation: symmetry methods and applications (Waterloo, ON, 1993), pages 45-66. Amer. Math. Soc., Providence, RI, 1996.

[4] H. S. Brown, I. G. Kevrekidis, and M. S. Jolly. A minimal model for spatiotemporal patterns in thin film flow. In Patterns and dynamics in reactive media (Minneapolis, MN, 1989), pages 11-31. Springer, New York, 1991.

[5] H.-C. Chang. Traveling waves on fluid interfaces: normal form analysis of the Kuramoto-Sivashinsky equation. Phys. Fluids, 29(10):3142-3147, 1986.

[6] H. Dankowicz, P. Holmes, G. Berkooz, and J. Elezgaray. Local models of spatio-temporally complex fields. Phys. D, 90(4):387-407, 1996.

[7] S. Dawson and A. Mancho. Collections of heteroclinic cycles in the Kuramoto-Sivashinsky equation. Phys. D, 100(3-4):231-256, 1997.

[8] E. Doedel. AUTO: a program for the automatic bifurcation analysis of autonomous systems. Cong. Num., 30:265-284, 1981.

[9] C. Foias, M. Jolly, I. Kevrekidis, G. Sell, and E. Titi. On the computation of inertial manifolds. Phys. Lett. A, 131(7-8):433-436, 1988.

[10] C. Foiaş, B. Nicolaenko, G. R. Sell, and R. Temam. Inertial manifolds for the Kuramoto-Sivashinsky equation and an estimate of their lowest dimension. J. Math. Pures Appl. (9), 67(3):197-226, 1988.

[11] R. W. Ghrist, P. J. Holmes, and M. C. Sullivan. Knots and links in threedimensional flows. Springer-Verlag, Berlin, 1997.

[12] P. Glendinning and C. Sparrow. Local and global behavior near homoclinic orbits. J. Statist. Phys., 35(5-6):645-696, 1984.

[13] M. Golubitsky and D. Schaeffer. Singularities and groups in bifurcation theory. Vol. I, volume 51 of Applied Mathematical Sciences. Springer-Verlag, New York, 1985.

[14] M. Golubitsky, I. Stewart, and D. Schaeffer. Singularities and groups in bifurcation theory. Vol. II, volume 69 of Applied Mathematical Sciences. Springer-Verlag, New York, 1988. 
[15] B. Greenhill. Archaeology of the Boat. Wesleyan University Press, Middletown, Conn., 1976.

[16] J. M. Hyman and B. Nicolaenko. The Kuramoto-Sivashinsky equation: a bridge between PDEs and dynamical systems. Phys. D, 18(1-3):113-126, 1986. Solitons and coherent structures (Santa Barbara, Calif., 1985).

[17] M. Johnson. Computer-assisted studies and visualization of nonlinear phenomena: two-dimensional invariant manifolds, global bifurcations, and robustness of global attractors. Ph.D. dissertation, Princeton University, Program in Applied and Computational Mathematics, 1998.

[18] M. E. Johnson, M. S. Jolly, and I. G. Kevrekidis. Two-dimensional invariant manifolds and global bifurcations: some approximation and visualization studies. Numer. Algorithms, 14(1-3):125-140, 1997. Dynamical numerical analysis (Atlanta, GA, 1995).

[19] M. Jolly, I. Kevrekidis, and E. Titi. Approximate inertial manifolds for the Kuramoto-Sivashinsky equation: analysis and computations. Phys. D, 44(1-2):38-60, 1990.

[20] M.S. Jolly, R. Rosa, and R. Temam. Accurate computations on inertial manifolds (submitted). 1999.

[21] M.S. Jolly, R. Rosa, and R. Temam. Evaluating the dimension of an inertial manifold for the Kuramoto-Sivashinsky equation (in preparation). Advances in Differential Equations, 1999.

[22] P. Kent and J. Elgin. Travelling-waves of the Kuramoto-Sivashinsky equation: period-multiplying bifurcations. Nonlinearity, 5(4):899-919, 1992.

[23] I. Kevrekidis, B. Nicolaenko, and J. Scovel. Back in the saddle again: a computer assisted study of the Kuramoto-Sivashinsky equation. SIAM J. Appl. Math., 50(3):760-790, 1990.

[24] B. Krauskopf and H. Osinga. Two-dimensional global manifolds of vector fields. Chaos, 9(3):768-774, 1999.

[25] Y. Kuramoto and T. Tsuzuki. Persistent propagation of concentration waves in dissipative media far from thermal equilibrium. Prog. Theo. Phys., 55(2):356-369, 1976.

[26] X. Liu. Gevrey class regularity and approximate inertial manifolds for the Kuramoto-Sivashinsky equation. Phys. D, 50(1):135-151, 1991.

[27] E. Lorenz. Deterministic nonperiodic flows. J. Atmospheric Sci., 20:130$141,1963$.

[28] J. Palis, Jr. and W. de Melo. Geometric theory of dynamical systems. Springer-Verlag, New York-Berlin, 1982. 
[29] J.C. Robinson. Inertial manifolds for the Kuramoto-Sivashinsky equation. Phys. Lett. A, 184(2):190-193, 1994.

[30] R. Rosa. Approximate inertial manifolds of exponential order. Discrete Contin. Dynam. Systems, 1(3):421-448, 1995.

[31] J. Scovel, I. G. Kevrekidis, and B. Nicolaenko. Scaling laws and the prediction of bifurcations in systems modeling pattern formation. Phys. Letters A, 130:73-80, 1988.

[32] G. I. Sivashinsky. On flame propagation under conditions of stoichiometry. SIAM J. Appl. Math., 39(1):67-82, 1980.

[33] J. Swift and K. Weisenfeld. Supression of period doubling in symmetric systems. Phys. Rev. Letters, 52(9):705-708, 1984.

[34] R. Temam. Infinite-dimensional dynamical systems in mechanics and physics, volume 68 of Applied Mathematical Sciences. Springer-Verlag, New York, 1988.

[35] R. Temam and X. Wang. Estimates on the lowest dimension of inertial manifolds for the Kuramoto-Sivashinsky equation in the general case. Differential Integral Equations, 7(3-4):1095-1108, 1994.

[36] R. W. Wittenberg and P. Holmes. Scale and space localization in the Kuramoto-Sivashinsky equation. Chaos, 9(2):452-465, 1999. 

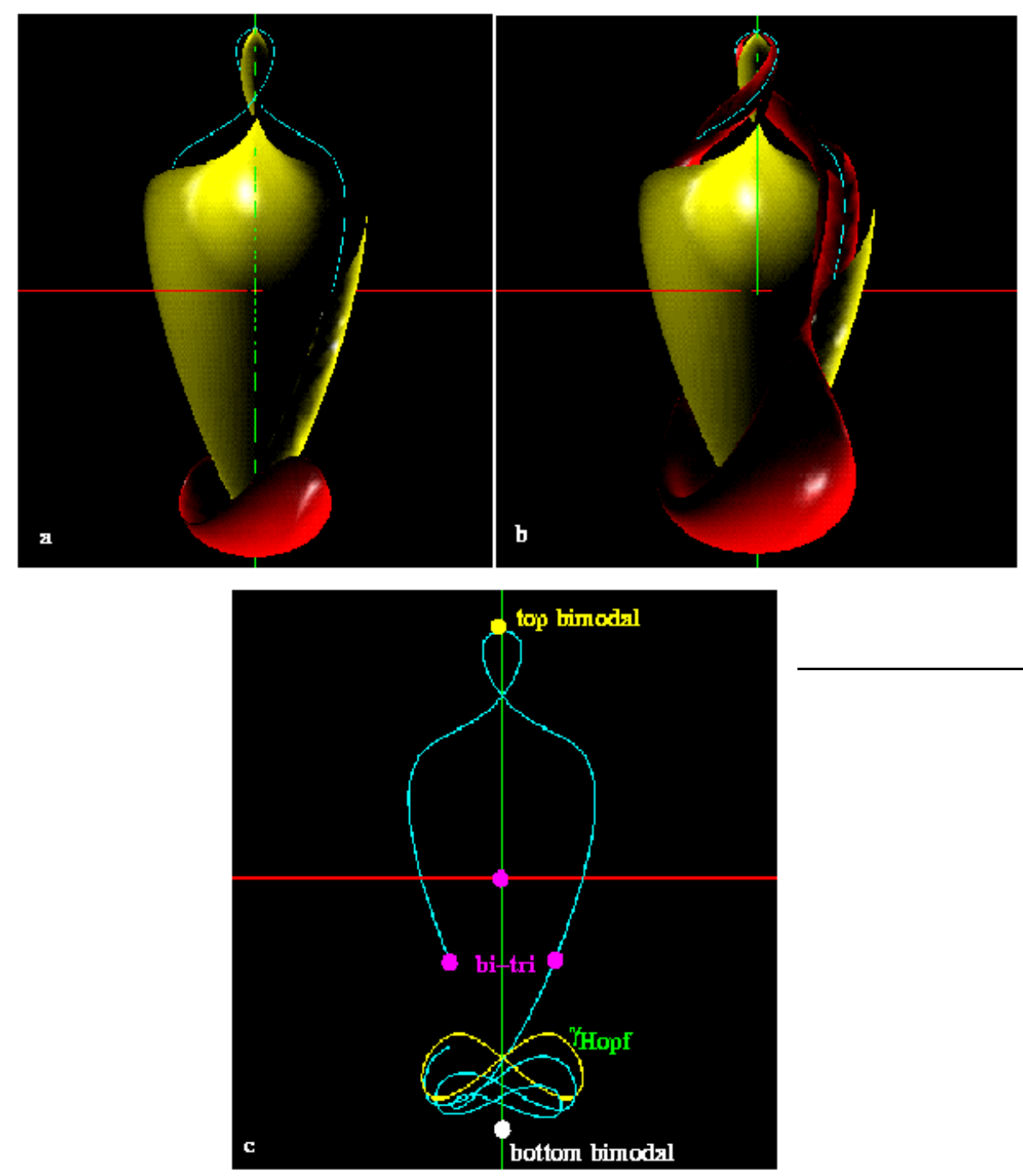

Figure 1: Elements of the global attractor for an eight-mode Galerkin approximation to the KSE. Shown in (a) and (b) are the two-dimensional unstable manifold of the origin (yellow), that of the bottom bimodal steady state (red) and the one-dimensional unstable manifolds of the bi-tris (blue), at two different values of the parameter: (a) $\alpha=32.0$ and (b) $\alpha=33.0$. (For an explanation of the steady state terminology see text). In (c), a skeleton of the global attractor is presented. 

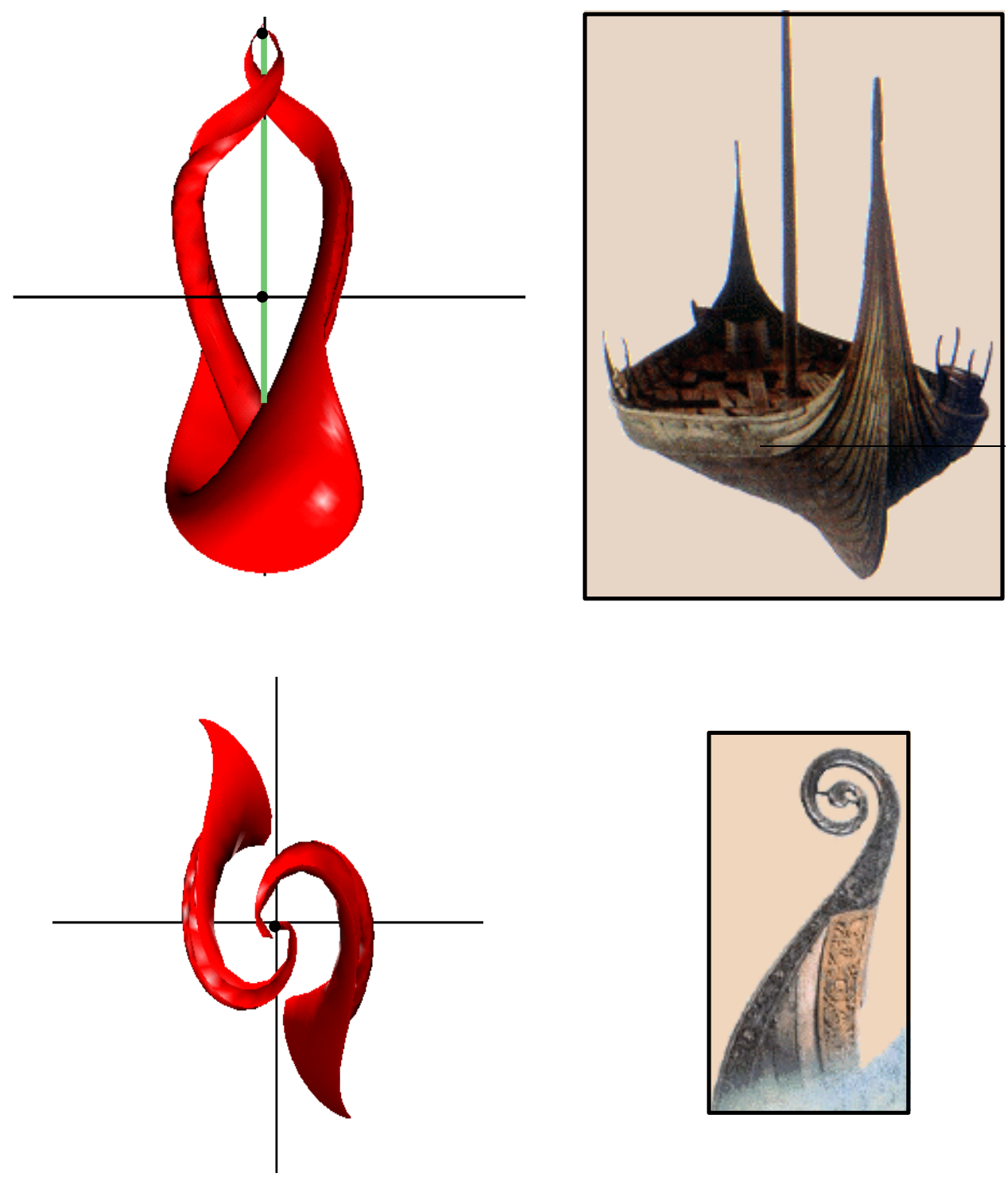

Figure 2: Separated at birth? The two-dimensional unstable manifold of the bottom bimodal (top: side view; bottom: top view) at $\alpha=33.0$ (see Figure 1) - the Oseberg ship. 


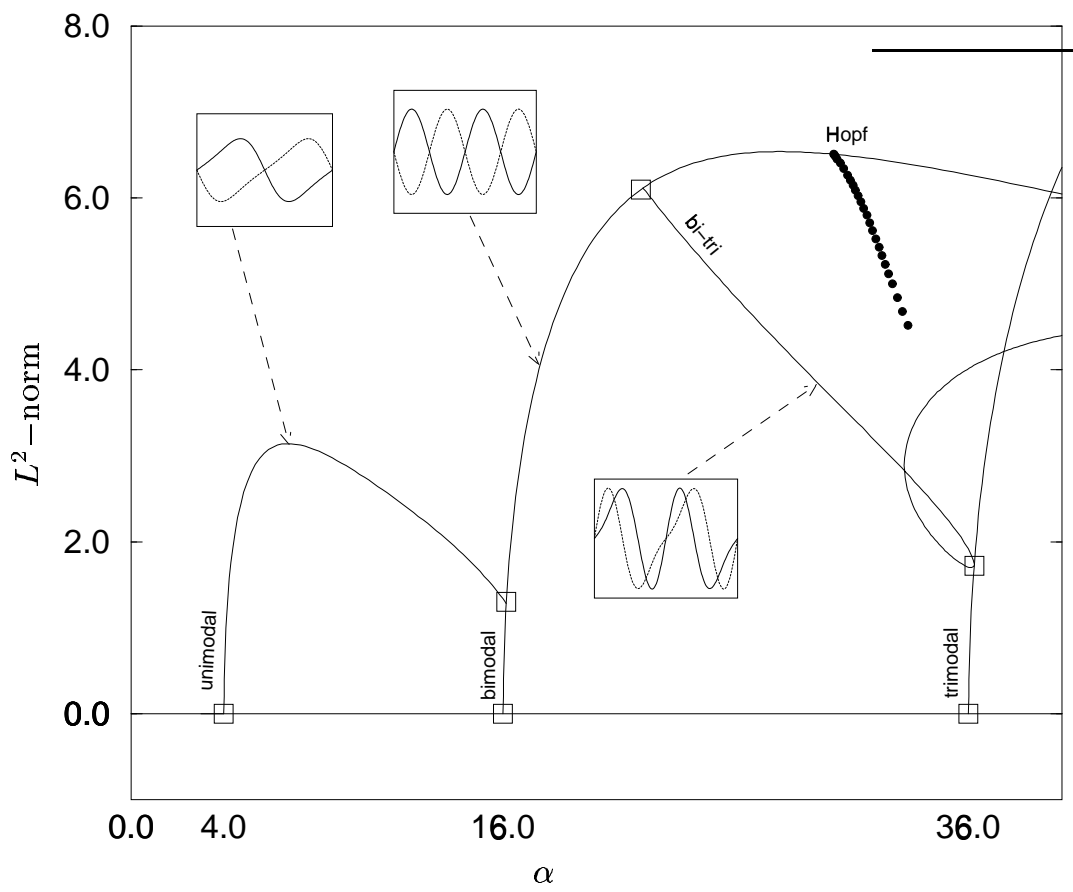

Figure 3: A view of the AIF bifurcation diagram showing various steady state branches as well as the onset of a relevant branch of oscillatory solutions. The insets illustrate the solution spatial profiles between zero and $2 \pi$. Representative phase portraits can be seen in Figure 4 . 


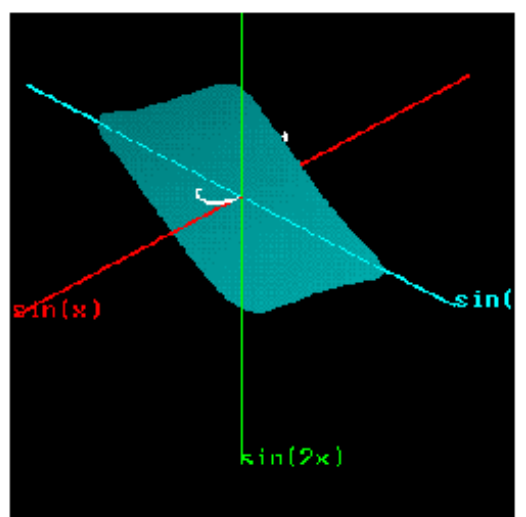

$\alpha=12.0$

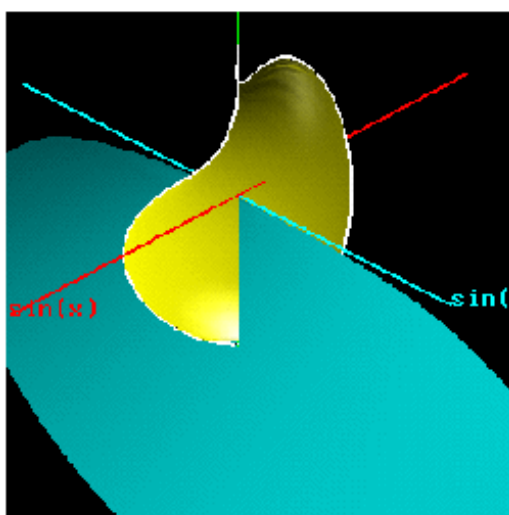

$\alpha=20.0$

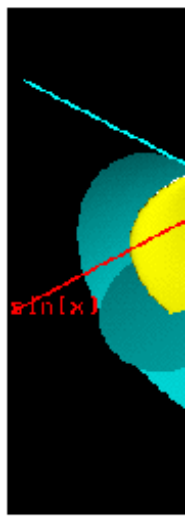

Figure 4: Representative phase portraits with unstable manifold of the origin in yellow, and one-dimensional unstable manifolds in white. The blue manifold is the two-dimensional stable manifold of, successively, the origin $(\alpha=12$, in (a)), the bottom bimodal steady state $(\alpha=20$, in (b)) and of the pair of bi-tri states $(\alpha=25$, in $(c))$. 

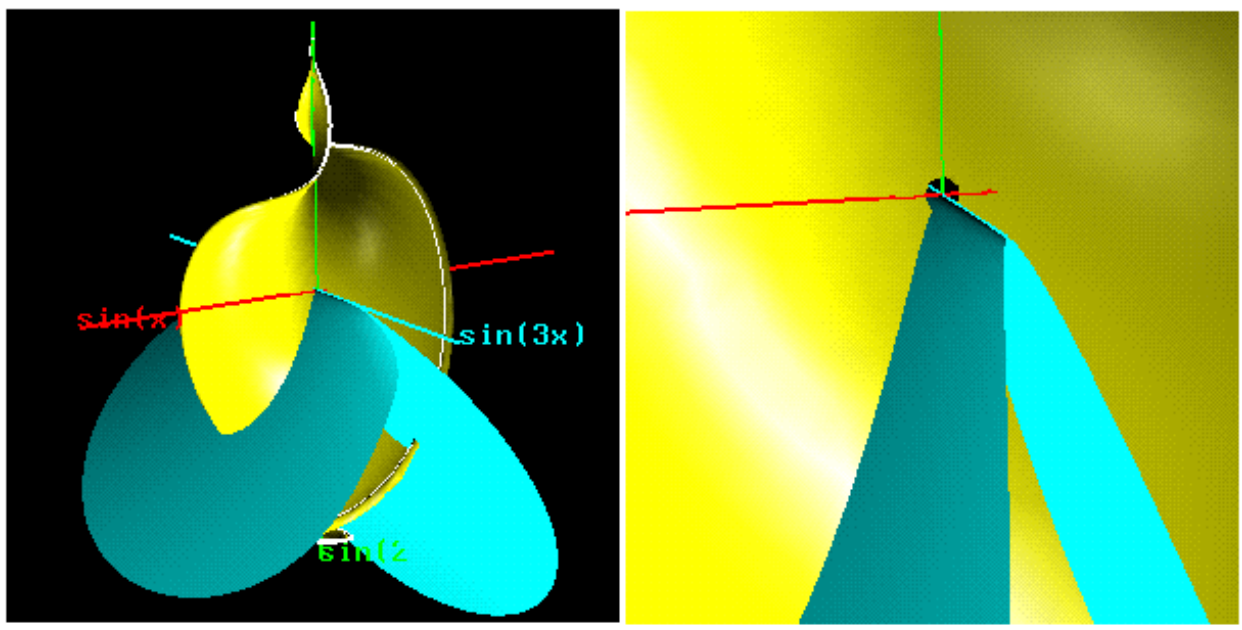

Figure 5: The tent (in blue) and the sail (in yellow) at $\alpha=27.0$. 

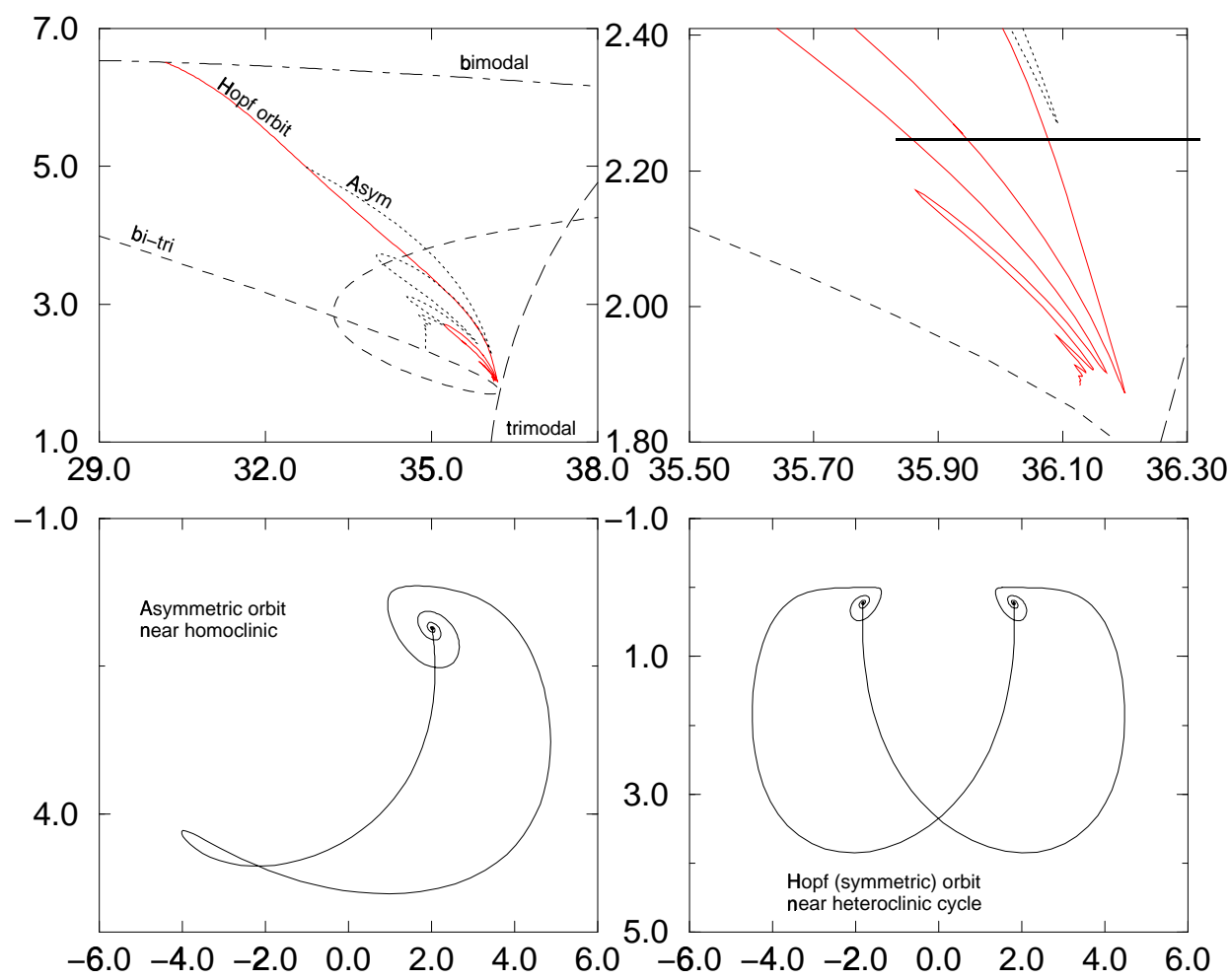

Figure 6: Limit cycle bifurcation diagram for the $\gamma_{\text {Hopf }}$ branch (see text). The representative phase plots illustrate the approach of the branch to the Silnikov homoclinic and heteroclinic connections involving the bi-tri steady states (for relatively large values of $\alpha$ ). The Hopf branch terminates in a Šilnikov connecting orbit at $\alpha \approx 36.12785$ (upper right); the asymmetric branch also terminates at $\alpha \approx 34.90108$ (upper left). The lower left frame shows a large-period, nearly homoclinic, asymmetric limit cycle at $\alpha=34.901079384$; the lower right shows a symmetric limit cycle near to a heteroclinic cycle at $\alpha=36.127432153$. 


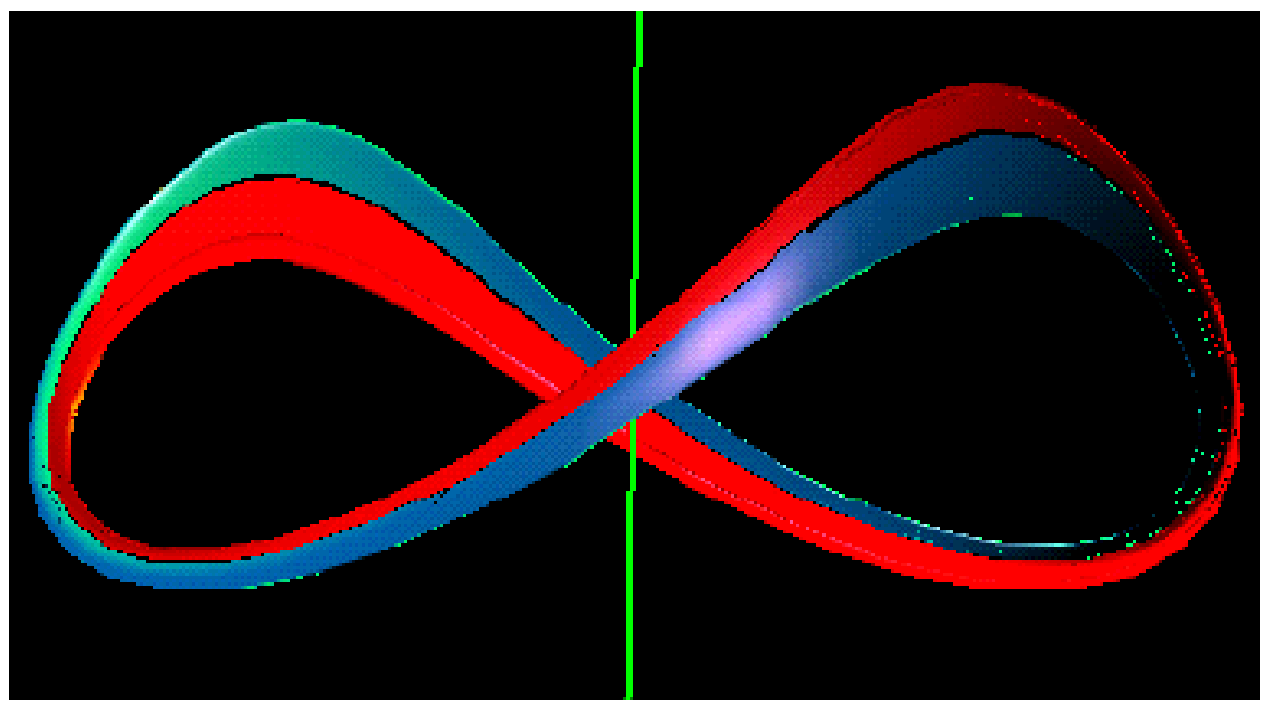

Figure 7: The two-dimensional unstable manifold of $\gamma_{\text {Hopf }}$ after the symmetry breaking bifurcation, one side in red, the other in blue. The green line is the $\sin (2 x)$ axis. The symmetric limit cycle lies at the boundary between the two sides (red and blue) of its unstable manifold; the asymmetric limit cycles constitute the edges of these two sides. 
$\alpha=32.62666$
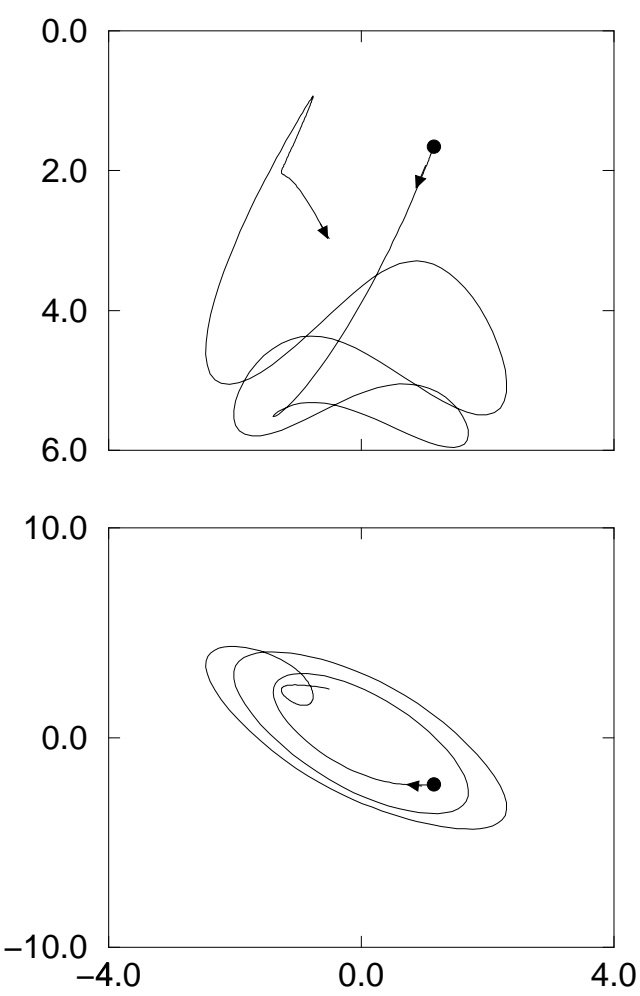

$\alpha=32.62667$
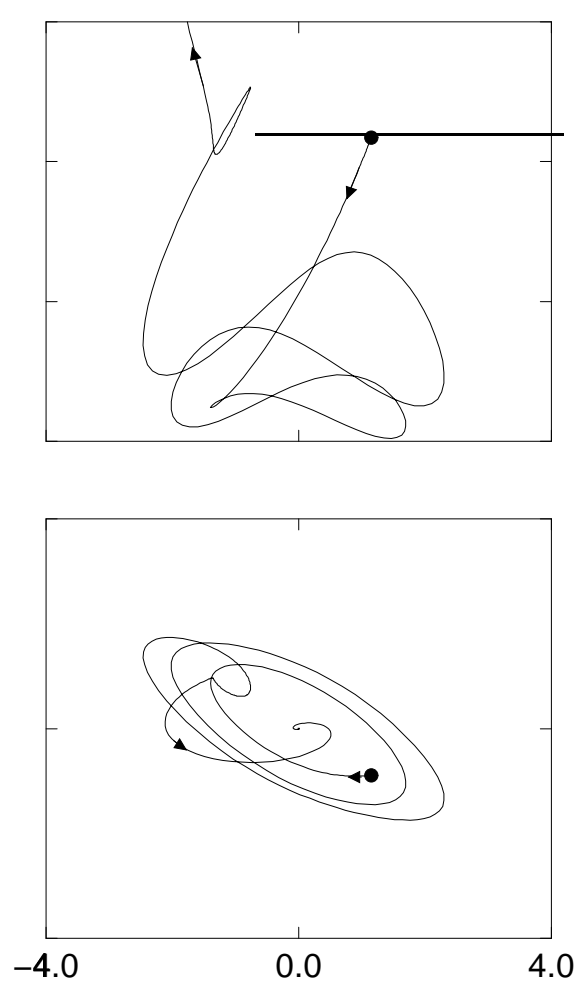

Figure 8: Two views (top: side view; bottom: top view) of the downward ropes (one-dimensional unstable manifolds of the bi-tri steady states); they are taken before (left) an after (right) a double heteroclinic connection. 


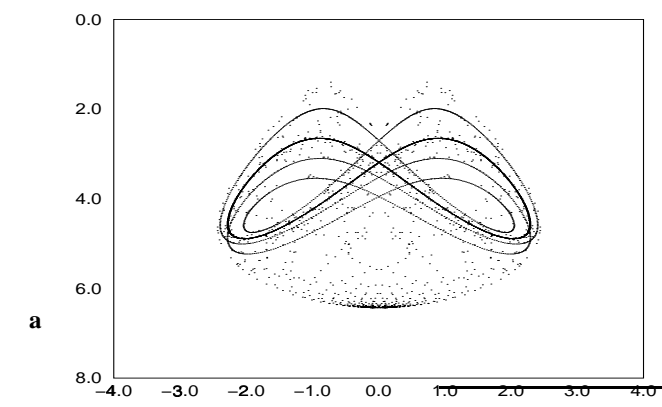

b
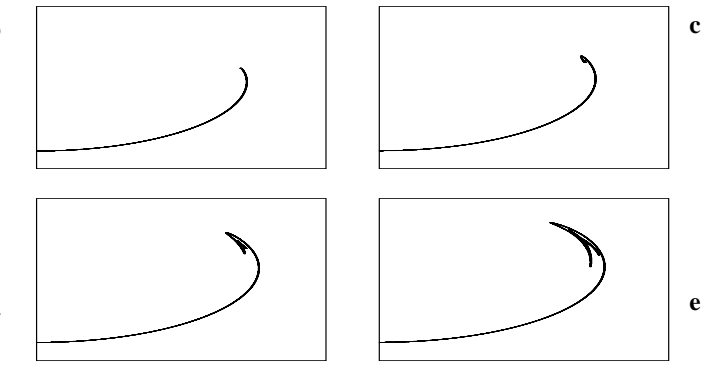

f
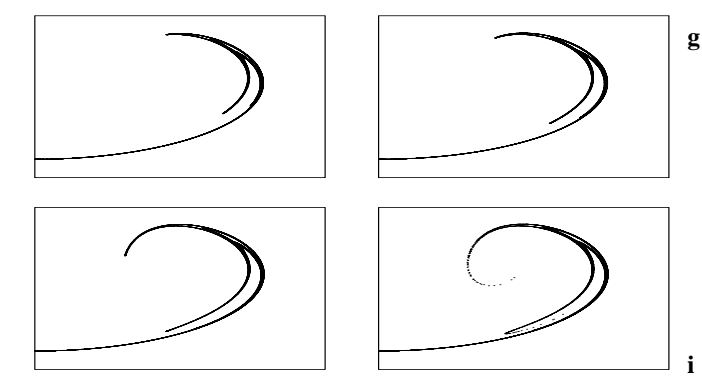

Figure 9: The hull lies in multiple basins of attraction at $\alpha=32.58$ (a); A oneparameter family of initial conditions on it (a circle of ICs close to the bottom bimodal) evolves (see the cloud of sampled transient points) in part to the symmetric $\gamma_{\text {Hopf }}$ and in part to a symmetric period-3 solution. The complexity of the hull increases with the parameter $\alpha$. We illustrate this through crosssection slices of the hull at various setting of $\alpha$, as follows: (b) 32.000, (c) 32.200 , (d) 32.400 , (e) 32.500 , (f) 32.573 , (g) 32.584, (h) 32.596, (i) 32.604. 

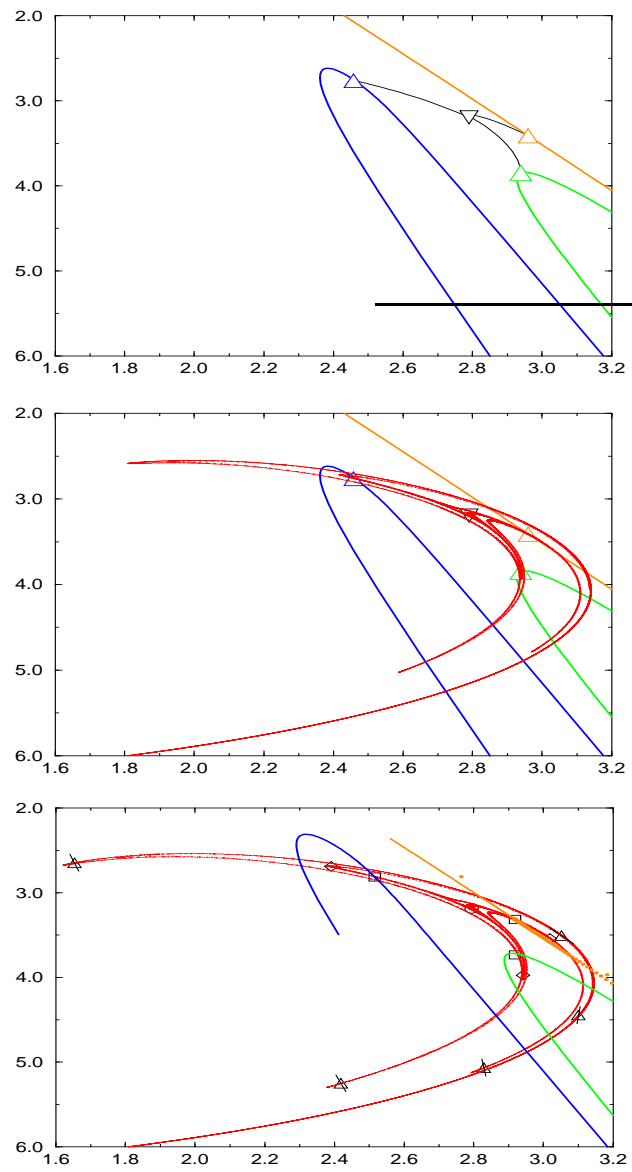

Figure 10: Within the Poincare section $b_{1}=0$, the three components of the stable manifold of the period-3 saddle limit cycle are plotted in blue, bronze, and green. In (a) and (b) the period-3 saddle is near its inception around $\alpha=32.57$, and labeled with $\Delta$, while $\gamma_{\text {Hopf }}$ is labeled with $\nabla$. The cross-section of the hull is shown in red in both (b) and (c). In (c), for slightly larger $\alpha$, a period- 5 saddle, denoted by $\Delta$, is present near the tips of the hull. The period-3 saddle and node in (c) are labeled by a square and a diamond respectively. The black curves are respectively, the unstable manifold of the period-3, in (a) and (b), and a small portion of that for the period-5 in (c). See text for discussion. 


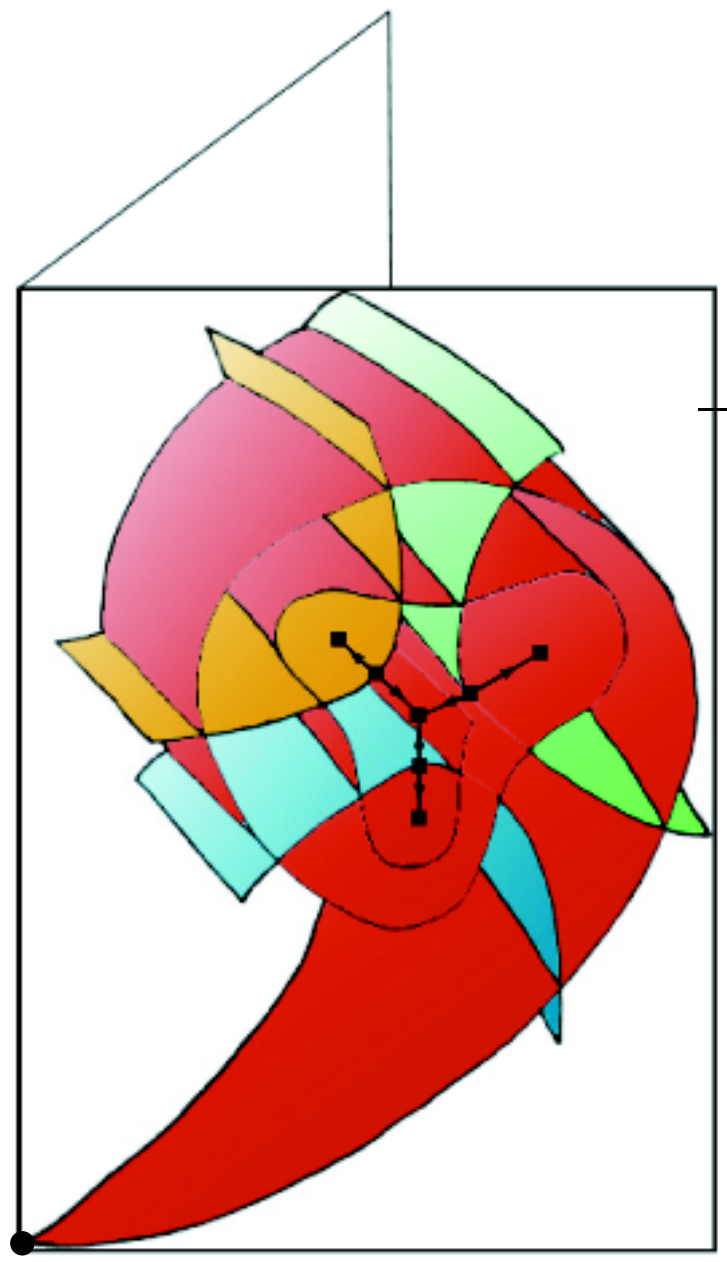

Figure 11: A schematic version of a portion of the hull, along with the stable manifolds of the period-3 saddle limit cycle, as they might appear trapped between two Poincaré sections. The colors are meant to be consistent with those in Figure 10 

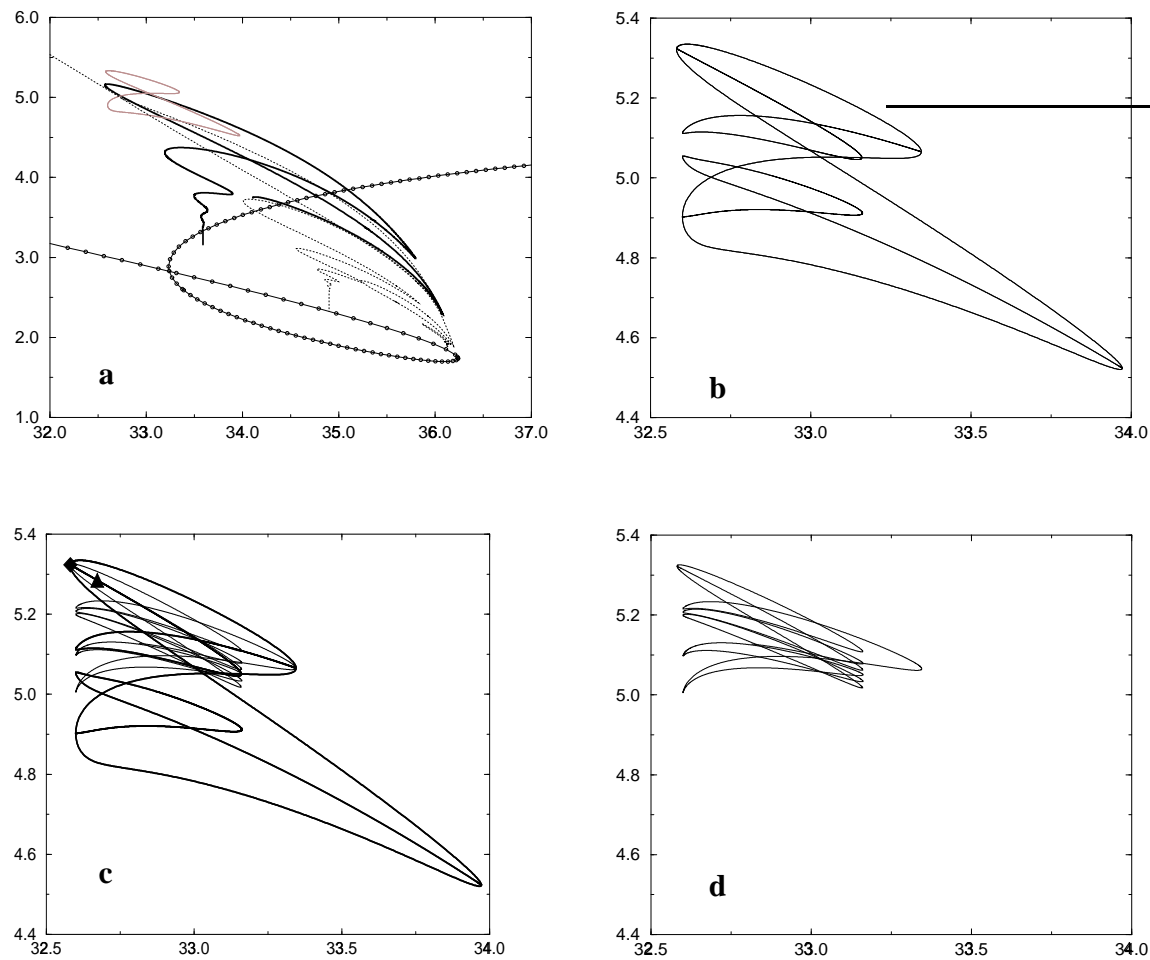

Figure 12: Bifurcation diagrams. (a) bi-tri steady state (solid with dots), period3 (bold, solid), Hopf (- - ) and symmetric period-5 (- - - - ), (b) blow-up showing only symmetric period- 5 from (a) along with asymmetric period- 5 , (c) same as (b) with a asymmetric period-10 branch, superposed, (d) period-10 branch alone on same scale. In (a) the period-3 branch ends in an Silnikov connection at $\approx 33.5903$, the other Śilnikov connections are at 34.9028 and 36.1263 . 
m
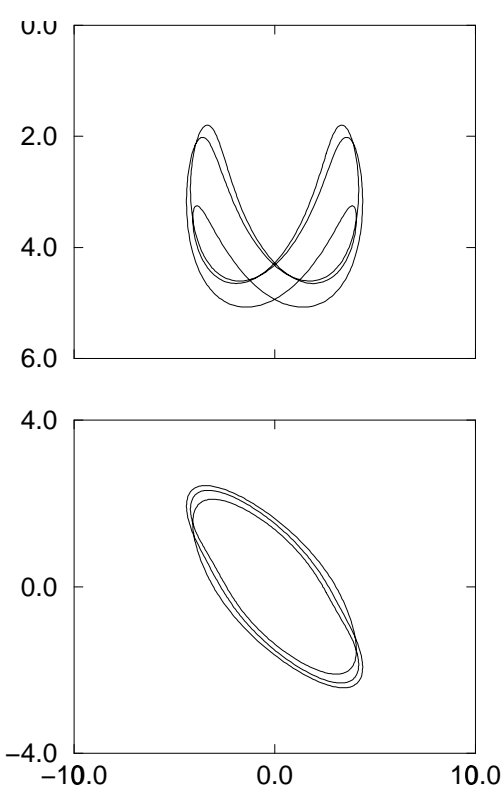

$\alpha=32.5818$
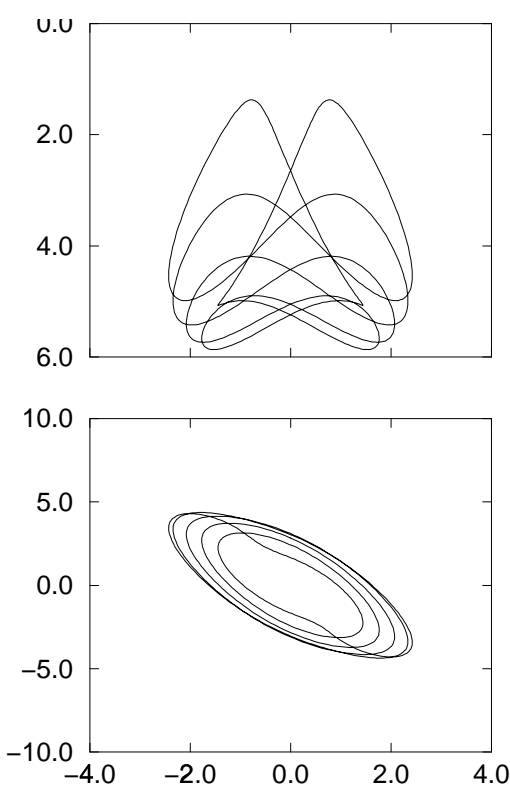

m
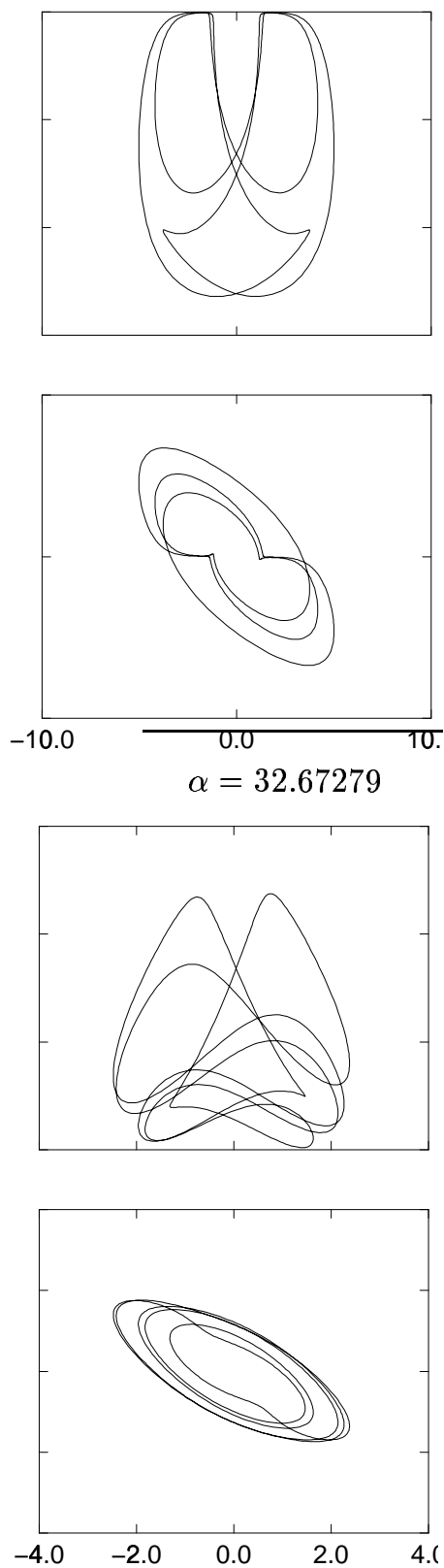

Figure 13: Representative phase portraits of the period-3 and period-5 limit cycles in the bifurcation diagrams in Figure 12 (in each case, top: side view; bottom: top view). 


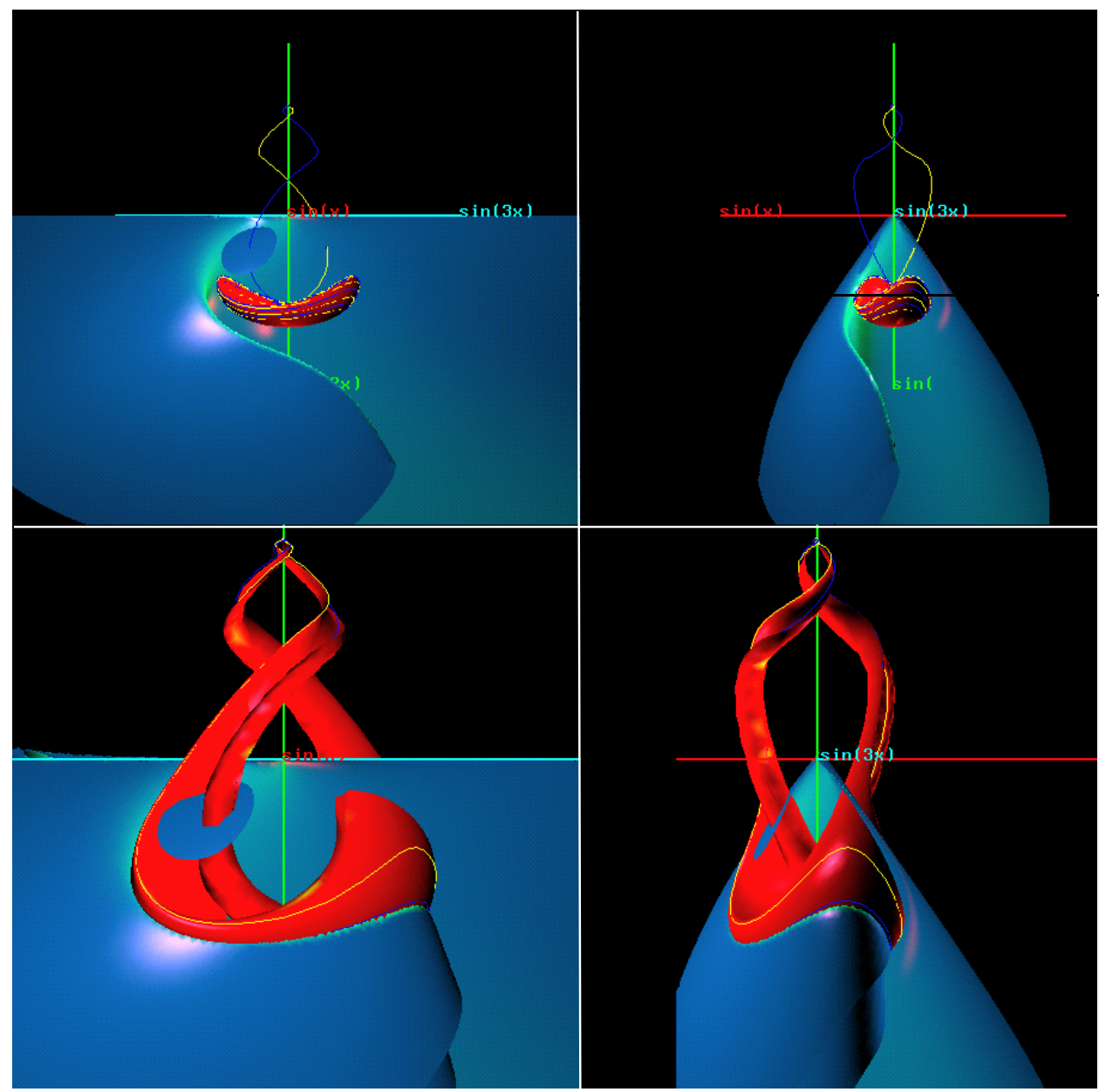

Figure 14: The Oseberg transition for the AIF (7) viewed from afar. The top two pictures are of phase space at $\alpha=32.0$ and the bottom two are from $\alpha=32.8$. The hull is in red, the tent in blue; one rope is yellow, the other blue. See text for detailed description. 

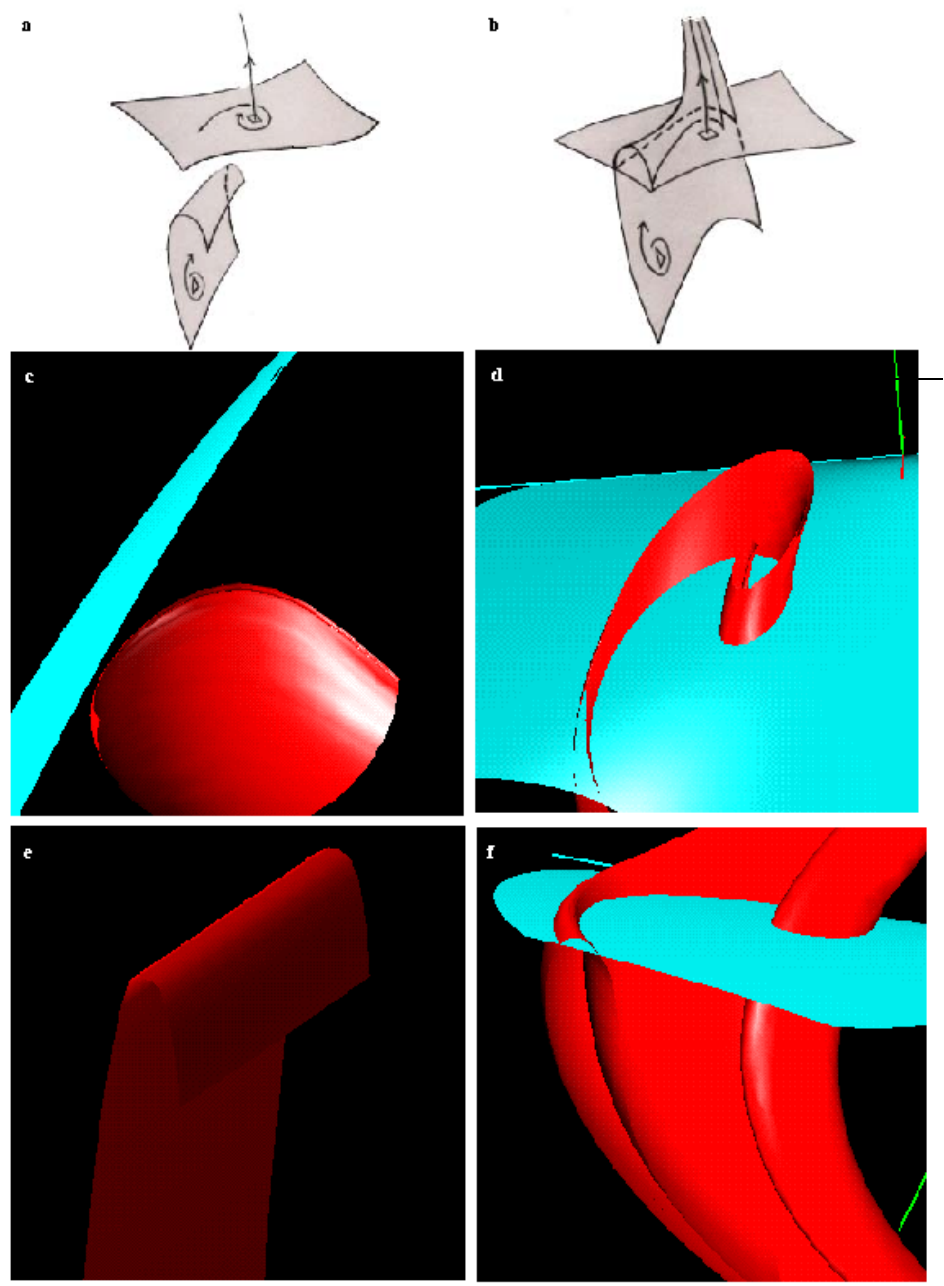

Figure 15: Schematic versions of the hull and tent near the bi-tri steady state are shown in (a) the dinghy regime, (b) the Oseberg ship regime; the small square represents the bi-tri state, and the small triangle, the bottom bimodal. Computational versions of the same transition follow in (c-f). In (c) a portion of the hull (red) and one side of the tent (blue) are constructed for $\alpha=32.0$, while in (e) a closeup of the rim of the hull is shown at the same parameter value. Two views of the Oseberg ship near a bi-tri state for $\alpha=32.8$ are shown in (d) and (f). The intersection of the two two-dimensional manifolds is a stable heteroclinic connection between the bottom bimodal and the bi-tri steady states. 